\title{
RESEARCH ON MANUFACTURING RESOURCE DISCOVERY AND SCHEDULING IN MANUFACTURING GRID
}

\author{
Yu'an $\mathrm{He}^{1,2}$, Tao $\mathrm{Yu}^{1}$, Lilan Liu ${ }^{1}$, Haiyang Sun ' \\ 1.Cims \& Robot Center, Shanghai University, 200072 China. 2.Shanghai Institute of \\ Technology, China 200233 Email: hya@graduate.shu.edu.cn
}

Abstract: The significant goal of Manufacturing Grid (MG) is to realize resource sharing and collaboration working among the heterogeneous and geographical distributed manufacturing resources. A TQPS (Time, Quality, Price, Service) based resource discovery and scheduling algorithm is proposed in the paper. By the four processes of resource discovery, resource scheduling, dynamic negotiation and resource reservation, the optimal resource can be discovered and matched to the task/subtask. Under the precondition of assuring the Quality of Service (QoS), the searching and scheduling algorithm facilitates the realization for the resource discovery and scheduling.

Key words: Manufacturing Grid (MG); Resource management; Resource discovery; Resource scheduling; Quality of Service (QoS).

\section{INTRODUCTION}

The goal of Manufacturing Grid (MG) ${ }^{1}$ is to realize manufacturing resource sharing and collaboration working. When $M G$ receives a manufacturing task, one of the significant functions is to search and schedule appropriate manufacturing resources across the different geographical distributed zones and enterprises, and effectively make these heterogeneous manufacturing resources sharing and collaboration. Because of Manufacturing resources' complexity and diversity, there are many differences between MG and computing grid in resource discovery and scheduling method, the resource management and scheduling methods in MG are more complicated than those in computing grid.

This paper is Supported by "SEC E-Institute: Shanghai High Institutions Grid" project \& "SEC project: No. 03HK07.

Please use the following format when citing this chapter:

$\mathrm{He}$, Yu'an, Yu, Tao, Liu, Lilan, Sun, Haiyang, 2006, in International Federation for Information Processing (IFIP), Volume 207, Knowledge Enterprise: Intelligent Strategies In Product Design, Manufacturing, and Management, eds. K. Wang, Kovacs G., Wozny M., Fang M., (Boston: Springer), pp. 902-907. 
The resource discovery and scheduling is a user-level middleware in fact. Document 2 proposed that MG system was a four-layer framework: 1) MG Fabric: this consists of all the globally distributed manufacturing resources that are accessible from anywhere on the Internet. 2) MG Core Middleware: this offers core services provided by GT4, such as security management, resource management, etc. 3) MG User-Level Middleware: this includes performance evaluating and monitoring tools, Manufacturing Grid Resource Scheduler (MGRS), etc. 4) MG Applications: MG applications are typically developed using Java language, e.g., MG Portal ${ }^{3}$.

Document 4 has ever proposed a QoS-based resource scheduling method, but when MG deals with complicated manufacturing tasks, resource consumers put forward more requirements for manufacturing service level and manufacturing resources, the search and scheduling method can not realize easily. In manufacturing, TQPS (Time, Quality, Price or Cost, Service) can describe a task's/subtask's requirements and a resource's characteristics well. So a new TQPS -based resource searching and scheduling algorithm is designed in the paper, this algorithm assures the resource consumers that they can obtain manufacturing services of quality assurance in $\mathrm{MG}$.

\section{THE PROCESS OF RESOURCE DISCOVERED AND SCHEDULING}

A manufacturing task or subtask decomposed by decomposing module must allocate an optimal resource which meets its QoS requirements. The process of searching and scheduling is shown in Fig.3.

Firstly, the matching resources are searched according to the task's QoS constraints, which include all the TQPS conditions, i.e., delivery time $\mathrm{T}$, task's quality $\mathrm{Q}$, task's price $\mathrm{P}$ and service requirements $\mathrm{S}$. Comparing the task's TQPS constraints with resources' TQCS properties, i.e., resource spare time $T$, resource service quality $Q$, resource cost $C$ and resource service $\mathrm{S}$, a resource list can be obtained if there are matching resources discovered. Then an optimal resource can be matched to the task according to Optimal Evaluation Model (OEM) ${ }^{4}$.

Secondly, considering the particularities of manufacturing resources, for the time $\mathrm{T}$, resource providers can compensate the using time for working overtime; for the price $\mathrm{P}$, resource consumers can consult or negotiate with the resource providers. So if all the TQPS constraints do not meet all the resource TQCS properties, the task's Q and S constraints are compared with resources' $\mathrm{Q}$ and $\mathrm{S}$ properties. If there are matching resources discovered, 
then $\mathrm{T}$ and $\mathrm{P}$ constraints are matched with $\mathrm{T}$ and $\mathrm{C}$ properties successively, or consumers can consult or negotiate with resource providers, then a resource list is also gained.

Thirdly, a Service Level Agreement (SLA) is subscribed by the MG-QoS manager, resources reservation is settled, and workflow model is formed.

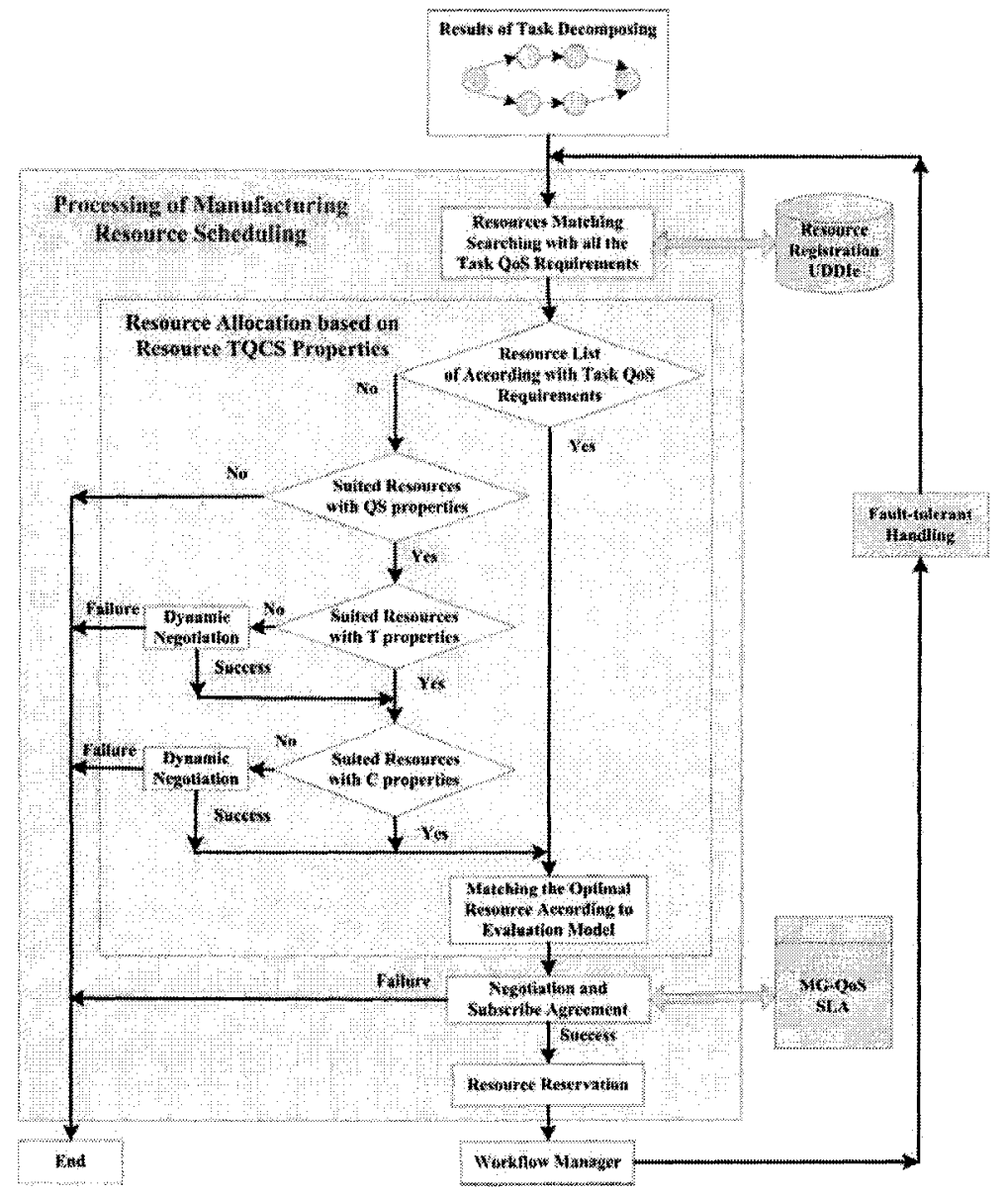

Figure 1. The process of resource discovery and scheduling

In addition, sometimes the SLA can not be carried out for some reasons, e.g., the reason of the resource fault, this time the matching resource must be searched and scheduled again through fault-tolerant handling.

In other word, there are four main steps in this process: TQPS-based Resource Discovery, TQPS-based Resource Scheduling, Dynamic Negotiation and Resource Reservation. 
Document 5 presented three types discovery methods: 1) Gold level: The value of resources' QoS attributes should not less than that the customer specified task's QoS constraints. 2) Silver level: the value of resources' QoS attributes could be varied within a range according to the task's QoS constraints. 3) Bronze level: customers just care about one or some QoS constraints, such as delivery time, product quality, cost and service, etc. According to the manufacturing particularities, resource discovery strategy in $\mathrm{MG}$ is always bronze level, so the discovery method can not operate and realize easily.

We presented a new algorithm using all TQCS properties or part of them, i.e., if no one resource is discovered using all task's TQPS constraints, then using $\mathrm{Q}$ and $\mathrm{S}$ constrains searching resources, if a resource list is gained, for the $T$ and $P$ constrains, the resource consumer can negotiate dynamically with resource providers. This method not only accords with the manufacturing facts, but also operates and realizes easily.

\section{TQPS-BASED ALGORITHM OF RESOURCE DISCOVERY AND SCHEDULING}

The searching process takes resource static QoS attributes (using mainly the TQPS) as restriction conditions, i.e., resource spare time $T$, service quality $\mathrm{Q}$, cost $\mathrm{C}$ and attributes related to service $\mathrm{S}$, to search and discover matching resources from MGIS (Manufacturing Resource Information System). Since UDDIe (Universal Description, Discovery and Integration Extension) provides the Application Programming Interface (API, i.e., the find_service) can realize resource discoveries which are different QoS attributes.

STEP1: constructing the task's subsets and QoS constraints. For a task submitted to MG is $T=\left\{t_{i}\right\}, \quad(i=1,2, \cdots n), t_{i}$ is one of the subtasks which task $\mathrm{T}$ is decomposed. Its QoS constraints are $Q o S_{t i}=\left\{T_{s i}, T_{e i} Q_{i}, P_{i}, S_{i}\right\}$, $T_{s i}, T_{e i}$ are the start and end time of the task respectively; $Q_{i}$ is the quality requirements of the task; $P_{i}$ is the product price; $S_{i}$ is the service requirements of the task.

STEP2: constructing the resource subsets and QoS attributes. The resource subsets $R=\left\{r_{i}\right\}(j=1,2, \cdots m)$ are the resources which accord with the requirements of the task and come from all the resource nodes' MGIS. Its QoS attributes are $R Q O S_{j}=\left\{R T_{s j}, R T_{e j}, R Q_{j}, R C_{j}, R S_{j}\right\}, R T_{s j}, R T_{e j}$ are the start and end spare time of resources respectively; $R Q_{j}$ is the attributes related to quality; $R S_{j}$ is the attributes related to service. 
STEP3: searching resources according to all the task's QoS constraints. The matching resources $S R=\left\{R_{k}\right\},(k=1,2, \cdots r)$ must meet the following equation:

$$
\left\{\begin{array}{l}
T_{s} \geq R T_{T_{j}} T_{e_{i}} \leq R T_{e j} \\
Q_{i} \leq R Q_{j} \\
P_{i}<R C_{j} \\
S_{i} \leq R S
\end{array}\right.
$$

Where: the start time $T_{s i}$ of subtask $t_{i}$ is later than the start spare time $R T_{s j}$ of the resource $R_{k}$, and the end time $T_{e i}$ of subtask $t_{i}$ is earlier than the end spare time $R T_{e j}$ of the resource $R_{k}$. The restriction conditions $Q_{i}$ of the subtask $t_{i}$ are less than or equal to the quality levels $R Q_{j}$ which the resource $R_{k}$ can provide. The price restriction condition $P_{i}$ of the subtask $t_{i}$ is less than using cost $R C_{j}$ of the resource $R_{k}$. The service restriction conditions $S_{i}$ of the subtask $t_{i}$ are less than or equal to the service attributes' conditions $R S_{j}$ of the resource $R_{k}$.

STEP4: if there are resources discovered after STEP3's searching, then turning to STEP7 to begin resource scheduling, otherwise just using $Q_{i}$ and $S_{i}$ constraints of the subtask to match resources. That is, the matching resources $S R=\left\{R_{k}\right\}(k=1,2, \cdots r)$ must meet the following equation:

$$
\left\{\begin{array}{l}
Q_{i} \leq R Q_{j} \\
\widetilde{S}_{i} \leq R \widetilde{S}_{j}
\end{array}\right.
$$

The meanings of the variables are the same as equation (1).

STEP5: if there are still no resources discovered meeting $Q_{i}$ and $S_{i}$ constraints, then returning to the end. It is implied that no resource can undertake the subtask at present, otherwise turning to the STEP6.

STEP6: considering the manufacturing facts, resource providers can solve the problem of resource using time by working overtime, and consumers can negotiate with resource providers about production price. So if there are resources discovered meeting $Q_{i}$ and $S_{i}$ constraints of the subtask, then negotiating about the resource using time and production price successively. If the time and price are negotiated successfully, the resource $R_{k}$ is gained, otherwise returning to the end.

STEP7: according to the matching resources $\left\{R_{k}\right\}$ to choose the optimal resource with OEM. Its application process is as below:

Through this algorithm, manufacturing resources discovered can provide the best manufacturing services for the task in MG. 


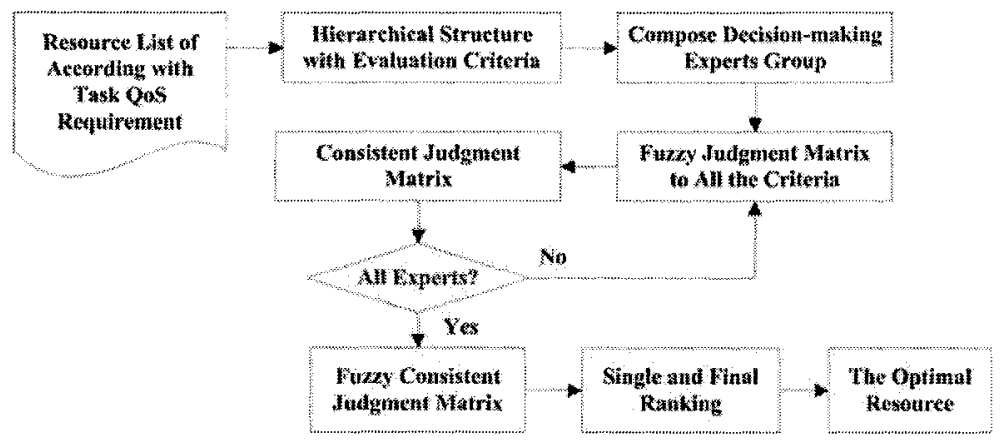

Figure 2. Application process of OEM

\section{CONCLUSION}

The discovery and scheduling of manufacturing resources were significant functions in MG. As the main requirements of manufacturing tasks and the properties of manufacturing resources, TQPS can describe a task's/subtask's constraints and a resource's characteristics well. In order to realize resource sharing and collaboration working among distributed manufacturing resources. A TQPS-based resource discovery and scheduling algorithm including dynamic negotiation and resource reservation can facilitate the realization for the resource discovery and scheduling.

\section{REFERENCES}

1. Liu Lilan, Yu Tao, Shi Zhanbei. Research of Self-Organization Manufacturing Grid and its Task Scheduling Algorithm, Computer integrated manufacturing system (CIMS), 2003, 9 (6): 449-455

2. He Yu'an, Yu Tao, Liu Lilan, et al. A QoS-based Task Decomposing Middleware Research for Manufacturing Grid. Proceedings of the First International Conference on Semantics, Knowledge and Grid, Beijing, China, 2005, 513-519

3. I. Foster, J. Frey, S. Graham, et al. "Open Grid Services Infrastructure (OGSI) Version 1.0", 2003, http://www.globus.org/research/papers.html.

4. Liu Lilan, Yu Tao, He Yu'an. A QoS-based GFAM Scheduling Approach for Manufacturing Grid. Proceedings of the Fifth International Conference on Computer and Information Technology (CIT'05). Shanghai, China, 2005,334-338

5. Liu Lilan, Yu Tao, Shi Zhanbei. Research on QoS-based Resource Scheduling in Manufacturing Grid, Computer Integrated Manufacturing System (CIMS). 2005, 11(4): pp $475-480$ 